\title{
Lista de Espécies de Borboletas (Lepidoptera: Hesperioidea e Papilionoidea) de uma Área de Semiárido na Região Nordeste do Brasil
}

\author{
José Nicássio Rocha Lima ${ }^{1} \bowtie$ Thamara Zacca ${ }^{2}$
}

1. Universidade do Estado da Bahia, e-mail: nikassiorocha@hotmail.com (Autor para correspondência ${ }^{\bowtie}$ ). 2. Universidade Federal do Paraná, e-mail: zacca.butterfly@gmail.com.

\section{EntomoBrasilis 7 (1): 33-40 (2014)}

Resumo. O Semiárido brasileiro corresponde, praticamente, aos limites do bioma Caatinga, sendo uma das regiões com menor número de unidades de conservação e com maior deficiência em estudos da biodiversidade de borboletas. Sendo assim, inventários visando contribuir para o conhecimento da diversidade local são extremamente necessários e urgentes. No presente estudo, uma lista das espécies de borboletas da Serra da Fumaça (Pindobaçu, Bahia, Brasil), foi elaborada baseada em amostragens mensais realizadas no período de Abril a Outubro de 2011, com duração de dois dias. Foram utilizadas rede entomológica e armadilhas com iscas de banana fermentada em caldo-de-cana. Em um total de 1.295 horas de amostragem (97 horas de captura com rede entomológica e 1.198 horas com armadilhas) foram registrados 756 indivíduos de 121 espécies, das quais dezessete são novos registros para o Semiárido e quatro, exclusivamente, para o estado da Bahia.

Palavras-chave: Bahia; Biodiversidade; Caatinga; Insecta; Inventário.

\section{List of Butterflies Species (Lepidoptera: Hesperioidea and Papilionoidea) in a Northeastern Brazilian Semiarid Area}

Abstract. The Brazilian Semiarid region practically corresponds to the Caatinga biome boundary, being one of the regions with lowest number of conservations unities and greater deficiency in butterflies' biodiversity studies. Therefore, inventories in order to contribute to knowledge of local diversity are extremely necessary and urgent. In the present study, a list of butterflies' species from Serra da Fumaça (municipality of Pindobaçu, state of Bahia, Brazil) was developed based on monthly sampling carried out from April to October 2011, lasting two days. The methods used were active capture with entomological nets and traps baited of fermented banana with juice sugar cane. In a total of 1,295 hours of sampling (97 hours of capture with entomological nets and 1,198 hours with traps) were registered 756 individuals of 121 species, of which seventeen are new records for the Semiarid region and four, exclusively, to state of Bahia.

Keywords: Bahia; Biodiversity; Caatinga; Insecta; Inventory.

$\Delta$ $\mathrm{s}$ poucas unidades de conservação existentes no Semiárido brasileiro e o fato destas apresentarem áreas bastante reduzidas são um desafio quanto à proteção dos ecossistemas regionais (MENDEs 1994). Áreas prioritárias para a conservação da biodiversidade no bioma Caatinga foram estabelecidas durante o Seminário de Planejamento Ecorregional da Caatinga (Velloso et al. 2002), mas a falta de inventários e dados taxonômicos confiáveis ainda é um dos grandes fatores que dificulta a criação de novas unidades de conservação (РPBıo 2011). Neste contexto, estudos que visam contribuir para o conhecimento da biodiversidade no Semiárido são extremamente necessários e urgentes.

De acordo com SANTos et al. (2008), o Semiárido representa uma das grandes lacunas no conhecimento da biodiversidade de borboletas, reflexo do baixo número de especialistas trabalhando nesta região. O primeiro inventário foi realizado por NoBRE et al. (2008), no Parque Nacional do Catimbau, Pernambuco, com registro de 121 espécies. Em seguida, ZACCA (2009) lista as espécies depositadas no museu da Universidade Estadual de Feira de Santana, Bahia, destacando registros novos para o Semiárido. Em um estudo realizado por PALUch et al. (2011) no Parque Ecológico João Vasconcelos Sobrinho, Pernambuco, foram registradas 197 espécies de borboletas, sendo a segunda maior lista para a região. Uma lista é apresentada por ZACCA et al. (2011), com registro de 140 espécies em uma área de ecótono no Semiárido baiano. Um estudo sobre as borboletas frugívoras do Parque Nacional do Catimbau foi realizado por Nobre et al. (2012), complementando o trabalho anterior. Posteriormente, ZACCA \& BRAVO (2012) realizaram inventários algumas localidades consideradas prioritárias para a conservação da fauna e flora pelo MMA (2007), situadas na porção norte da Chapada Diamantina, com ocorrência de 169 espécies e vários novos registros para o estado.

No presente trabalho é apresentada uma lista das espécies de borboletas da Serra da Fumaça, município de Pindobaçu, Bahia, com objetivo de gerar informações básicas para eventuais planos de conservação e manejo local, bem como contribuir para o conhecimento da diversidade de borboletas no Semiárido brasileiro e no estado da Bahia.

\section{MATERIAL E MÉTODOS}

A Serra de Jacobina é um complexo montanhoso localizado na meso-região centro-oeste do estado da Bahia, com $220 \mathrm{~km}$ de extensão no sentido norte-sul, variando de 6 a $10 \mathrm{~km}$ no sentido leste-oeste (Velloso et al. 2002; VAle 2005). Constituise de escarpas abruptas, vales profundos, "canyons" cortados por rios, córregos e riachos, que originam quedas d'águas e 
T cachoeiras (VAle 2005). Apesar de inclusa no bioma Caatinga, estudo realizado por Ramalho (2008) indica que o domínio da Serra de Jacobina possui um clima tipicamente sub-úmido, com temperatura média anual de $24^{\circ} \mathrm{C}$, pluviosidade variando entre 477,6 e $1.129,3 \mathrm{~mm} /$ ano (VALE 2005) e períodos secos e chuvosos bem definidos, de maio a setembro e de outubro a abril, respectivamente (VelLoso et al. 2002). A Serra de Jacobina está inserida na área de Senhor do Bonfim, sendo de importância extremamente alta para conservação, devido à grande diversidade fisionômica, além de conter espécies da fauna e flora ameaçadas de extinção e endêmicas (Velloso et al. 2002; MMA 2007; PPBıo 2011).

Dentre as cadeias montanhosas deste complexo, destaca-se a Serra da Fumaça $\left(10^{\circ} 39^{\prime} \mathrm{S} 40^{\circ} 22^{\prime} \mathrm{W}\right)$, localizada entre os municípios de Antônio Gonçalves, Senhor do Bonfim e Pindobaçu, este último, onde as amostragens foram realizadas. Possui 4.000 hectares, com ascensões variando entre $400 \mathrm{~m}$ e $1.020 \mathrm{~m}$ de altitude, nos quais podem ser encontradas formações vegetais bem diversificadas como Floresta Estacional Semidecidual (Figura 1A), Matas de Grotões, Cerrado, Campo Sujo e Campo Rupestre (Figura $1 \mathrm{~B}$ ), predominante na localidade.

As amostragens foram realizadas entre abril e outubro de 2011, com duração de dois dias em cada mês. Um transecto de aproximadamente $4 \mathrm{~km}$ foi estabelecido, sendo percorrido uma vez por dia de coleta, iniciando-se na base da serra, em uma área de Floresta Estacional Semidecidual, e terminando no topo (Figura 2). A captura dos indivíduos foi realizada com rede entomológica, por um único coletor, e nove armadilhas com isca de banana fermentada com caldo de cana, dispostas em pontos predeterminados, com altura variando entre 1-2 m em relação ao solo, com distância mínima de $300 \mathrm{~m}$ entre cada. A instalação das armadilhas foi realizada à medida que o transecto era percorrido no primeiro dia de cada coleta e, sua remoção e dos exemplares capturados, no dia seguinte. Para determinação do tempo de amostragem, cada armadilha foi considerada como uma unidade amostral, sendo efetuada a soma dos períodos que cada uma ficou ativa durante as sete coletas.

O material coletado encontra-se depositado nas coleções entomológicas da Universidade Estadual da Bahia (UNEB) e Prof. Johann Becker do Museu de Zoologia da Universidade Estadual de Feira de Santana (MZUEFS). A identificação taxonômica segue a classificação proposta por LAMAs (2004).

Para elaboração da curva de acumulação de espécies foi utilizado o programa EstimateS v. 8.2 (Cowell 2006), com os estimadores de riqueza não paramétricos Jackknife 1 e Bootstrap. A confirmação de novos registros de borboletas para o Semiárido, bem como para o estado da Bahia foi realizada por meio de consulta aos seguintes trabalhos: MAY (1924); NoBRE
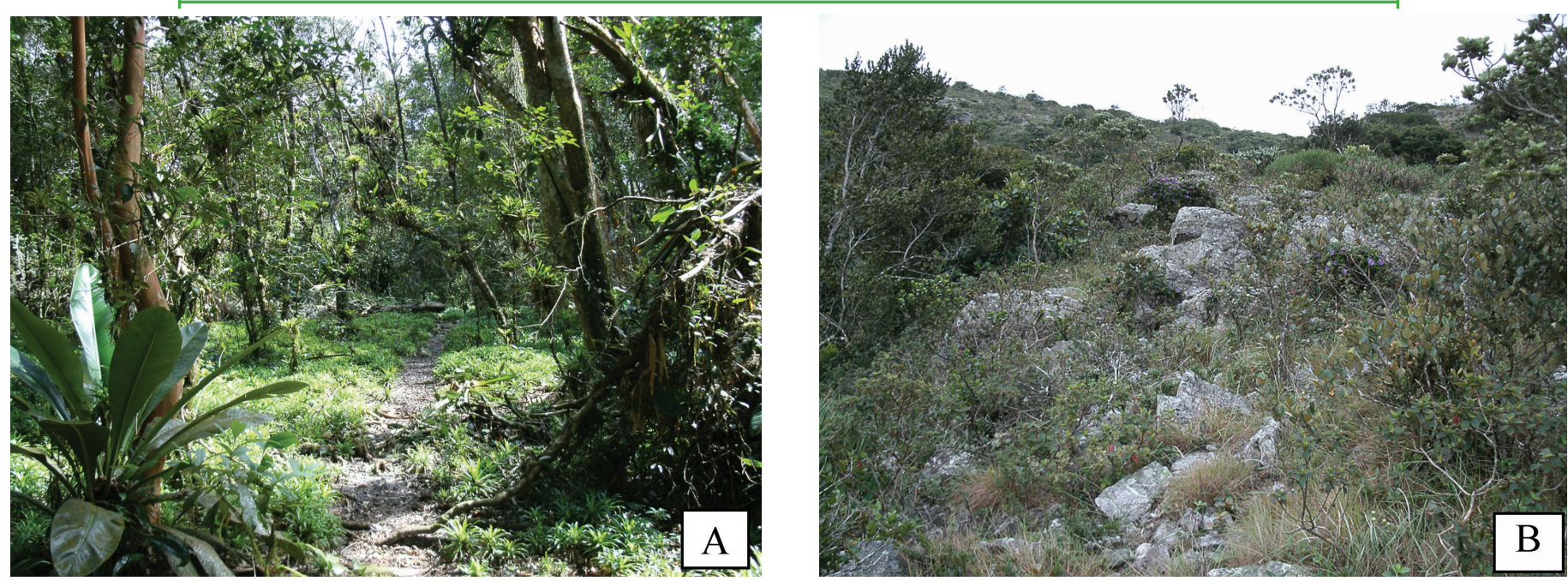

Figura 1. Fitofisionomias da Serra da Fumaça: (A) Floresta Estacional Semidecidual; (B) Campo Rupestre.

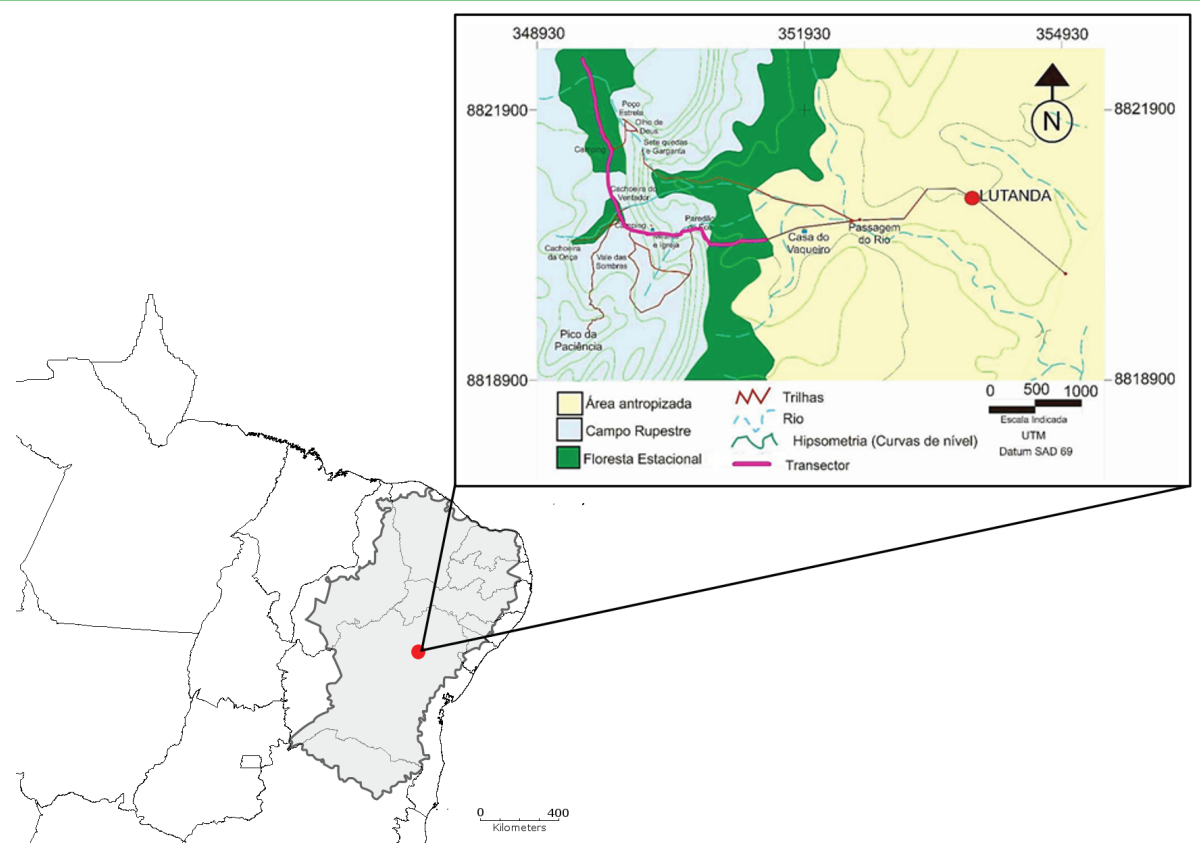

Figura 2. Mapa representando a delimitação do Semiárido brasileiro (área em cinza) e localização da Serra da Fumaça (ponto vermelho), com ênfase na distribuição de suas principais fitofisionomias, trilhas e transecto percorrido. (Modificado de Rangel B. Carvalho). 
et al. (2008); VAsCONCElos et al. (2009); ZACCA (2009); PALUCH et al. (2011); ZACCA et al. (2011); Nobre et al. (2012) e ZACCA \& BRAVO (2012). Comentários acerca da distribuição geográfica das espécies basearam-se em literatura disponível e no exame do material depositado na coleção entomológica Pe. Jesus Santiago Moure do Departamento de Zoologia da Universidade Federal do Paraná (DZUP)

\section{RESULTADOS E DISCUSSÃO}

Em um total de 1295 horas de amostragem (97 horas de captura com rede entomológica e 1198 horas com armadilhas), foram registrados 756 indivíduos de 121 espécies (Tabela 1), dos quais 40,5\% dos indivíduos foram capturados com rede (102 espécies) e $59,5 \%$ em armadilhas (24 espécies), sendo apenas cinco espécies comuns às duas metodologias. Nymphalidae apresentou maior diversidade quando somada as duas metodologias, com 54 espécies e 599 indivíduos, seguida de Hesperiidae (37 spp./ 95 ind.), Riodinidae (13 spp./ 27 ind.), Pieridae (9 spp./ 31 ind.), Lycaenidae (6 spp./ 13 ind.) e Papilionidae (2 spp./3 ind.).

Captura com armadilhas. Foram registradas cinco subfamílias de Nymphalidae. Biblidinae foi a mais representativa em termos de riqueza e abundância (11 espécies e 194 indivíduos), seguida por Satyrinae (6 spp. e 149 ind.), Charaxinae (4 spp. e 109 ind.), Nymphalinae (2 spp. e 4 ind.) e Morphinae (1 sp. e 1 ind.). De acordo com UEhara-Prado et al. (2007), as espécies de Biblidinae são favorecidas pela fragmentação ambiental, o que elucida o elevado número de espécies e indivíduos encontrados na área de Floresta Estacional limítrofe com a área antrópica da Serra da Fumaça.

As únicas publicações encontradas que apresentaram dados para captura com armadilhas atrativas, tanto no estado da Bahia quanto para o Semiárido, foram os de VASCONCELOS et al. (2009); Nobre et al. (2012) e ZACCA \& Bravo (2012). No entanto, este último apresenta dados permutados à metodologia de captura com rede entomológica. VASCONCELOS et al. (2009) apresenta um esforço amostral de 180 horas (estimativa ignora cada armadilha como unidade amostral, não havendo somatório de horas por armadilha, mas por coleta), em que foi possível observar uma riqueza similar a encontrada na Serra da Fumaça. Contudo, a abundância em ambos os estudos foi bem menor que a registrada no presente trabalho. Das espécies registradas por NoBre et al. (2012), apenas nove foram comuns à Serra da Fumaça, a citar: Biblis hyperia nectanabis (Frühstorfer), Callicore sorana sorana (Godart), Eunica tatila bellaria Frühstorfer, Hamadryas februa februa (Hübner), Hamadryas feronia feronia (Linnaeus), Fountainea halice moretta (Druce), Hypna clytemnestra forbesi (Godman \& Salvin), Melinaea ethra (Godart) e Taygetis laches (Fabricius).
Comparando os dados obtidos no presente estudo com os inventários realizados por Uehara-Prado et al. (2007); VASCONCELos et al. (2009); Roman et al. (2010) e Nobre et al. (2012), pode-se afirmar que a riqueza obtida foi satisfatória em relação ao tempo de amostragem. Contudo, é importante destacar que a amostragem com armadilhas atrativas restringiu-se a área de sub-bosque (altura máxima de $2 \mathrm{~m}$ em relação ao solo), privilegiando espécies que ocorrem neste ambiente. Estudos que visaram analisar a estratificação vertical de borboletas frugívoras (DeVRIEs et al. 1997; DeVRIES \& Walla 2001; Roman et al. 2010) indicam variação quanto a riqueza de espécies entre ambientes de sub-bosque e dossel. Sendo assim, presume-se que a realização de amostragens no dossel possibilitaria um acréscimo da riqueza de borboletas frugívoras na Serra da Fumaça.

Captura com rede entomológica. Hesperiidae e Nymphalidae apresentaram maior número de espécies (37 spp. e 35 spp., respectivamente), seguida de Riodinidae (13 spp.), Pieridae (9 spp.), Lycaenidae (6 spp.) e Papilionidae (2 spp.). Quanto ao número de indivíduos registrados, Nymphalidae apresentou maior abundância com 142 indivíduos, seguida por Hesperiidae (95), Riodinidae (27), Pieridae (31), Lycaenidae (13) e Papilionidae (3).

O número pouco expressivo de Papilionidae na Serra da Fumaça e em demais localidades do Semiárido [ver Nobre et al. (2008) = 3 espécies; VAsCONCElos et al. $2009=3$ spp.; PAluch et al. (2011) = 4 spp.; ZaCCA et al. (2011) = 3 spp.; ZaCCA \& Bravo (2012) = 4 spp.], provavelmente está relacionado às reduzidas áreas de mata e carência de recursos hídricos nesta região já que, segundo BROWN JR. \& FreITAS (1999), as espécies desta família geralmente são encontradas em ambientes de matas preservadas e com abundância de recursos hídricos.

Inventários de borboletas realizados na região do Semiárido (Nobre et al. 2008; VASCONCELOS et al. 2009; PALUCH et al. 2011; ZACCA et al. 2011; ZACCA \& BRAVO 2012), obtiveram uma proporção diferenciada da riqueza encontrada no presente estudo e nos inventários em ambientes de Floresta Estacional (Mielke \& CASAGRANDE 1997; DessuY \& MoRAIS 2007), apontando Nymphalidae com maior número de espécies, seguida de Hesperiidae. Isto talvez se deva ao fato de Hesperiidae, juntamente com Lycaenidae e Riodinidae, serem consideradas de difícil amostragem e identificação (Brown JR. \& Freitas 1999; Freitas et al. 2003).

A curva acumulativa total de espécies (Figura 3) evidencia que a riqueza local de borboletas encontra-se subestimada e que, provavelmente, poderia aumentar com um maior esforço de amostragem. Os maiores valores de riqueza e abundância foram registrados no mês de Junho (Tabela 1), o que provavelmente está relacionado à expressiva disponibilidade de recursos florais,

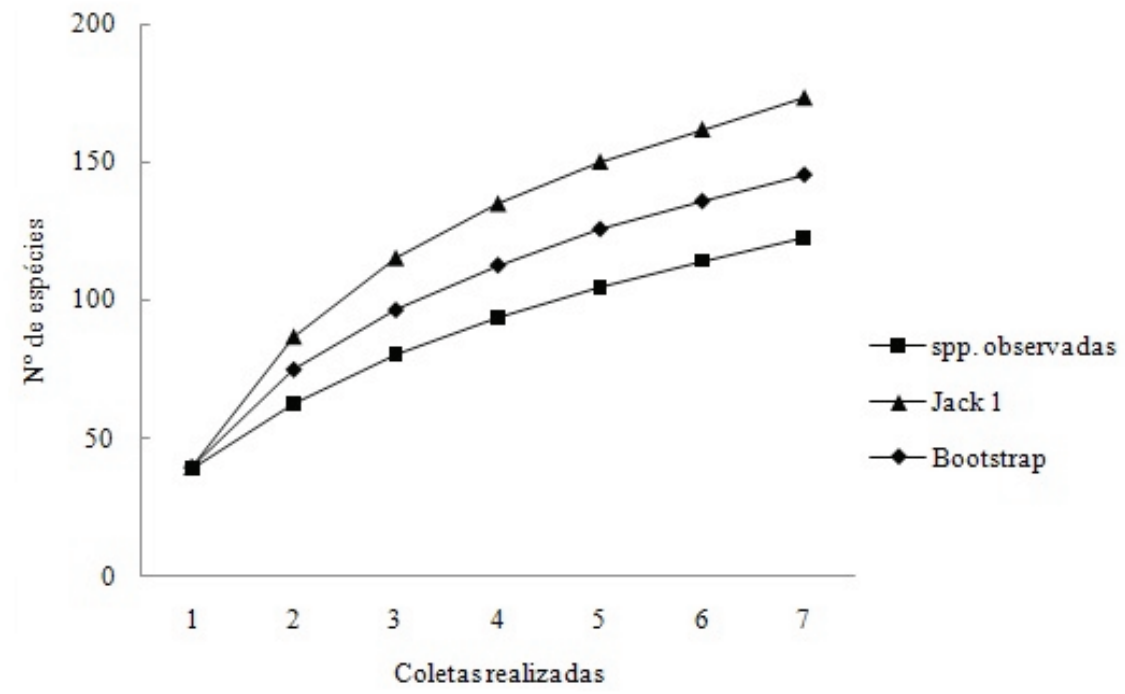

Figura 3. Curva de estimativa total de espécies de borboletas registradas para a Serra da Fumaça. 
Tabela 1. Lista das espécies de borboletas (Lepidoptera: Hesperioidea e Papilionoidea) da Serra da Fumaça, Pindobaçu, Bahia, Brasil, registradas no período de Abril a Outubro de 2011, com número de indivíduos por coleta, método de captura empregado (MC) e tipos de fitofisionomias amostradas (TFA): Campo Rupestre (CR), Floresta Estacional de baixa altitude (FEB) e Floresta Estacional de altitudes elevadas (FEA). Espécies registradas para o estado da Bahia por: "a" MAY (1924); "b" VASCONCElos et al. (2009); "c" ZACCA (2009); "d" ZACCA et al. (2011); "e" ZACCA \& BRAVO (2012). Novos registros para o Semiárido; * Novos registros para o estado da Bahia. $\mathrm{R}$ = rede entomológica; $\mathrm{A}$ = armadilha.

\begin{tabular}{|c|c|c|c|c|c|c|c|c|c|c|}
\hline \multirow{2}{*}{\multicolumn{2}{|c|}{ Táxon }} & \multirow{2}{*}{ TFA } & \multicolumn{7}{|c|}{ Indivíduos capturados por coleta } & \multirow{2}{*}{ MC } \\
\hline & & & Abr & Mai & Jun & Jul & Ago & Set & Out & \\
\hline \multicolumn{11}{|l|}{ Hesperidae } \\
\hline \multirow{13}{*}{ Hesperiinae } & Cobalopsis nero (Herrich-Schäffer) ${ }^{\mathbf{d}}$ & $\mathrm{CR}$ & 2 & - & - & - & - & - & - & $\mathrm{R}$ \\
\hline & Copaeodes jean favor Evans* & $\mathrm{CR}$ & 2 & - & - & - & - & - & - & $\mathrm{R}$ \\
\hline & Cumbre belli eberti Evans ${ }^{\text {e }}$ & $\mathrm{CR}$ & - & - & 1 & 1 & - & - & - & $\mathrm{R}$ \\
\hline & Cymaenes tripunctus theogenis (Capronnier) ${ }^{\mathrm{d}}$ & $\mathrm{CR}$ & - & 3 & - & 1 & - & - & - & $\mathrm{R}$ \\
\hline & Gesta gesta (Herrich-Schäffer) ${ }^{\mathbf{d}}$ & $\mathrm{CR}$ & - & - & - & 1 & - & - & - & $\mathrm{R}$ \\
\hline & Hylephila phyleus phyleus (Drury) ${ }^{\mathbf{b} \mathbf{d}}$ & $\mathrm{CR}$ & - & - & 1 & - & - & - & - & $\mathrm{R}$ \\
\hline & Niconiades xanthaphes Hübner, ${ }^{\mathbf{e}}$ & FEA & - & - & 1 & - & - & - & - & $\mathrm{R}$ \\
\hline & Orses cynisca (Swainson,) ${ }^{* *}$ & $\mathrm{CR}$ & - & - & - & - & 1 & 1 & - & $\mathrm{R}$ \\
\hline & Panoquina l. lucas (Fabricius) d e & $\mathrm{CR}$ & - & - & 2 & - & - & - & - & $\mathrm{R}$ \\
\hline & Panoquina peraea (Hewitson) •* & $\mathrm{CR}$ & - & - & 3 & - & - & 1 & - & $\mathrm{R}$ \\
\hline & Pompeius pompeius (Latreille) ${ }^{\mathbf{b} \mathbf{e}}$ & CR/FEA & - & - & 4 & - & 1 & - & 1 & $\mathrm{R}$ \\
\hline & Saliana longirostris (Sepp)* & $\mathrm{CR}$ & - & - & 3 & 1 & - & - & - & $\mathrm{R}$ \\
\hline & Vettius lafrenaye lafrenaye (Latreille) d e & FEA & - & - & - & 1 & - & - & - & $\mathrm{R}$ \\
\hline \multirow{24}{*}{ Pyrginae } & Aguna asander asander (Hewitson) ${ }^{\mathrm{d}}$ & $\mathrm{FEB} / \mathrm{CR}$ & - & 1 & 3 & - & 1 & 1 & - & $\mathrm{R}$ \\
\hline & Anastrus sempiternus simplicior (Möschler) ${ }^{\mathbf{d}}$ & FEA & - & - & - & 1 & - & - & - & $\mathrm{R}$ \\
\hline & Astraptes fulgerator fulgerator (Walch) ${ }^{*}$ & FEA & - & 1 & - & - & - & - & - & $\mathrm{R}$ \\
\hline & Autochton integrifascia (Mabille) •* & $\mathrm{CR}$ & - & 1 & - & - & - & - & - & $\mathrm{R}$ \\
\hline & Chioides catillus (Cramer)* & $\mathrm{FEA} / \mathrm{CR}$ & 1 & - & 4 & - & - & 1 & 1 & $\mathrm{R}$ \\
\hline & Chiomara georgina georgina (Reakirt) ${ }^{* *}$ & CR & - & - & 2 & - & - & - & - & $\mathrm{R}$ \\
\hline & Cogia calchas (Herrich-Schäffer) ${ }^{\mathbf{c}}$ & FEA & - & - & - & - & 1 & - & - & $\mathrm{R}$ \\
\hline & Epargyreus socus (Hübner)* & CR/FEA & - & 2 & - & - & 1 & - & 1 & $\mathrm{R}$ \\
\hline & Gorgythion plautia (Möschler) d e & CR & - & - & - & 1 & - & - & - & $\mathrm{R}$ \\
\hline & Gorgythion sp. & $\mathrm{CR}$ & - & 1 & - & - & - & - & - & $\mathrm{R}$ \\
\hline & Heliopetes arsalte arsalte (Linnaeus) ${ }^{\mathbf{b} \mathbf{c d ~} \mathbf{e}}$ & $\mathrm{CR}$ & - & - & 1 & - & - & - & - & $\mathrm{R}$ \\
\hline & Heliopetes alana (Reakirt) $\mathbf{d}$ & $\mathrm{CR}$ & - & - & - & - & 1 & - & - & $\mathrm{R}$ \\
\hline & Narcosius parisi parisi (Williams) ${ }^{*^{*}}$ & $\mathrm{CR}$ & - & - & - & - & 1 & - & - & $\mathrm{R}$ \\
\hline & Pyrgus orcus (Stoll) d e & $\mathrm{CR}$ & - & - & - & - & - & - & 1 & $\mathrm{R}$ \\
\hline & Staphylus sp. & $\mathrm{CR}$ & 5 & 1 & 1 & - & - & - & - & $\mathrm{R}$ \\
\hline & Udranomia spitzi (Hayward) •* & CR & 1 & - & - & - & - & - & - & $\mathrm{R}$ \\
\hline & Urbanus chalco (Hübner) d e & FEA & - & 1 & - & 1 & - & - & - & $\mathrm{R}$ \\
\hline & Urbanus dorantes dorantes (Stoll) d e & $\mathrm{FEB} / \mathrm{CR}$ & 1 & 1 & 3 & 3 & 3 & - & - & $\mathrm{R}$ \\
\hline & Urbanus procne (Plötz) ${ }^{\mathrm{e}}$ & $\mathrm{FEB} / \mathrm{CR}$ & - & 4 & 1 & 1 & 1 & - & - & $\mathrm{R}$ \\
\hline & Urbanus proteus proteus (Linnaeus) ${ }^{\mathbf{d} \mathbf{e}}$ & CR & - & 1 & 1 & - & - & - & - & $\mathrm{R}$ \\
\hline & Urbanus simplicius (Stoll) ${ }^{\mathbf{b} \mathbf{d}}$ & CR & 1 & - & - & - & - & - & - & $\mathrm{R}$ \\
\hline & Urbanus teleus (Hübner) ${ }^{\mathbf{b} \mathbf{d}}$ & $\mathrm{CR}$ & - & - & - & - & 1 & - & - & $\mathrm{R}$ \\
\hline & Urbanus virescens (Mabille) ${ }^{\mathrm{d}}$ & FEB & - & - & - & - & 1 & - & - & $\mathrm{R}$ \\
\hline & Xenophanes tryxus (Stoll) • & $\mathrm{CR}$ & - & - & - & 1 & - & - & - & $\mathrm{R}$ \\
\hline
\end{tabular}

\section{Papilionidae}

$\begin{array}{lllllllll}\mathrm{CR} & - & - & 2 & - & - & - & - & \mathrm{R} \\ \mathrm{CR} & - & - & 1 & - & - & - & - & \mathrm{R}\end{array}$

\section{Pieridae}


Tabela 1. Continuação...

\begin{tabular}{|c|c|c|c|c|c|c|c|c|c|c|}
\hline & \multirow{2}{*}{ Táxon } & \multirow{2}{*}{ TFA } & \multicolumn{7}{|c|}{ Indivíduos capturados por coleta } & \multirow{2}{*}{ MC } \\
\hline & & & Abr & Mai & Jun & Jul & Ago & Set & Out & \\
\hline \multirow{4}{*}{ Coliadinae } & Phoebis philea philea (Linnaeus) ${ }^{\mathbf{c d}}$ & $\mathrm{CR}$ & - & - & 1 & - & - & - & - & $\mathrm{R}$ \\
\hline & Phoebis sennae marcellina (Cramer) ${ }^{\mathbf{b} \mathbf{c d e}}$ & $\mathrm{CR}$ & 1 & - & 1 & - & 1 & - & - & $\mathrm{R}$ \\
\hline & Pyrisitia leuce leuce (Boiduval) $^{\mathrm{d} \text { e }}$ & CR/FEA & - & - & 1 & 1 & - & - & 1 & $\mathrm{R}$ \\
\hline & Pyrisitia nise tenella (Boisduval) ${ }^{\mathrm{d}} \mathrm{e}$ & $\mathrm{CR}$ & 1 & 1 & - & - & - & - & 1 & $\mathrm{R}$ \\
\hline Pierinae & Ascia monuste orseis (Godart) b $\mathbf{c} \mathbf{d e}$ & $\mathrm{CR}$ & - & - & - & - & - & 1 & - & $\mathrm{R}$ \\
\hline \multicolumn{11}{|l|}{ Lycaenidae } \\
\hline Polyommatinae & Leptotes cassius cassius (Cramer) ${ }^{\mathbf{b} \mathbf{c} \mathbf{d} \mathbf{e}}$ & $\mathrm{CR}$ & - & - & 1 & - & - & - & 1 & $\mathrm{R}$ \\
\hline \multirow{5}{*}{ Theclinae } & Pseudolycaena marsyas (Linnaeus) b c e & FEA & - & - & 1 & 1 & - & - & - & $\mathrm{R}$ \\
\hline & Strymon bubastus bubastus (Stoll) d e & $\mathrm{CR}$ & - & - & 1 & - & - & - & - & $\mathrm{R}$ \\
\hline & Strymon rufofusca (Hewitson) ${ }^{\mathbf{d} \mathbf{e}}$ & CR/FEA & - & 1 & 1 & - & - & - & - & $\mathrm{R}$ \\
\hline & Strymon crambusa (Hewitson)* & CR & - & - & - & - & 1 & - & - & $\mathrm{R}$ \\
\hline & Theritas hemon (Cramer) ${ }^{\mathrm{d} \mathbf{e}}$ & FEA & - & 1 & 1 & - & - & 3 & - & $\mathrm{R}$ \\
\hline \multicolumn{11}{|l|}{ Riodinidae } \\
\hline Euselasiinae & Euselasea thucydides thucydides (Fabricius) $\mathrm{e}$ & $\mathrm{CR}$ & 2 & - & - & - & - & - & - & $\mathrm{R}$ \\
\hline \multirow{11}{*}{ Riodininae } & Aricoris sp. & $\mathrm{CR}$ & 1 & - & - & - & - & - & - & $\mathrm{R}$ \\
\hline & Echydna chaseba (Hewitson) d e & FEB & - & - & - & 2 & - & - & - & $\mathrm{R}$ \\
\hline & Emesis fatimella fatimella Westwood ${ }^{*}$ & FEB & 1 & - & - & - & - & - & - & $\mathrm{R}$ \\
\hline & Ionotus alector (Geyer)* & CR/FEA & - & 2 & - & - & - & - & - & $\mathrm{R}$ \\
\hline & Isapis agyrtus (Cramer)* & CR & - & - & - & - & - & 1 & - & $\mathrm{R}$ \\
\hline & Juditha molpe (Hübner) ${ }^{\mathrm{d}}$ & FEA & 1 & - & - & 1 & - & - & - & $\mathrm{R}$ \\
\hline & Leucochimona icare matatha (Hewitson) ${ }^{\mathrm{d}} \mathbf{e}$ & FEB/FEA & - & 1 & - & 1 & 1 & 1 & - & $\mathrm{R}$ \\
\hline & Melanis xenia xenia (Hewitson) ${ }^{\mathbf{c e}}$ & $\mathrm{FEB} / \mathrm{CR}$ & - & 2 & - & 1 & - & 1 & - & $\mathrm{R}$ \\
\hline & Nymphidium azanoides Butler $\mathbf{e}$ & FEA & - & - & 1 & - & - & - & - & $\mathrm{R}$ \\
\hline & Nymphidium lisimon attenuatum Stichel ${ }^{* *}$ & $\mathrm{CR}$ & 1 & - & - & - & - & - & - & $\mathrm{R}$ \\
\hline & Synargis galena (Bates) ** & $\mathrm{CR}$ & 3 & - & 2 & 1 & - & - & - & $\mathrm{R}$ \\
\hline
\end{tabular}

\section{Nymphalidae}

Biblidinae

Charaxinae

Danainae

Heliconiinae

\section{Biblis hyperia nectanabis (Fruhstorfer) ${ }^{\mathbf{b} \mathbf{c d} \mathbf{e}}$ \\ Callicore sorana sorana (Godart) ce \\ Eunica macris macris (Godart) ${ }^{\mathrm{e}}$ \\ Eunica tatila bellaria Fruhstofer ${ }^{\mathbf{b}} \mathbf{c} \mathbf{e}$ \\ Eunica eurota dolores (Prittwitz)** \\ Hamadryas amphinome amphinome \\ (Linnaeus) $^{\mathbf{b} \mathbf{c d e}}$ \\ Hamadryas arete (Doubleday) ${ }^{\mathbf{e}}$}

Hamadryas arinome obnubila (Fruhstorfer)

•*

Hamadryas epinome (C. Felder \& R. Felder) ${ }^{\text {e }}$

Hamadryas februa februa (Hübner) ${ }^{\mathbf{e}}$

Hamadryas feronia feronia (Linnaeus) ${ }^{\mathbf{b} \text { e }}$

Hamadryas iphthime iphthime (Bates) ${ }^{\mathbf{e}}$

Marpesia chiron marius (Cramer) ${ }^{\mathbf{c d}}$

Mestra hersilia hypermnestra Hübner ${ }^{\mathrm{cd}}$

Fountainea glycerium cratais (Hewitson) ${ }^{\mathbf{e}}$

Fountainea halice moretta (Druce) ${ }^{\mathbf{c}}$

Hypna clytemnesta forbesi Godman \& Salvin

ce

Siderone galanthis galanthis (Cramer) ${ }^{\bullet}$

Danaus erippus (Cramer) ${ }^{\mathbf{c}}$

Danaus gilippus gilippus (Cramer) ${ }^{\mathbf{e}}$

Lycorea halia discreta Haensch c d e

Actinote genitrix genitrix D'Almeida*

Agraulis vanilae maculosa (Stichel) ${ }^{\mathbf{b}} \mathbf{c d e}$

Eueides isabella dianasa (Stoll) ${ }^{\mathbf{a}} \mathbf{b} \mathbf{c d} \mathbf{e}$

\begin{tabular}{|c|c|c|c|c|c|c|c|c|}
\hline CR/FEA & - & - & 1 & 1 & - & 2 & - & $\mathrm{R} / \mathrm{A}$ \\
\hline CR & - & - & - & - & - & 1 & 1 & A \\
\hline $\begin{array}{c}\text { FEB/FEA/ } \\
\text { CR }\end{array}$ & 3 & 12 & 6 & 1 & - & 3 & 12 & $\mathrm{R} / \mathrm{A}$ \\
\hline FEB/CR & - & - & 1 & - & - & 2 & - & A \\
\hline FEA & - & - & - & 1 & - & - & - & $\mathrm{R}$ \\
\hline FEB & 1 & - & - & - & - & - & 1 & A \\
\hline FEB & 6 & - & - & - & - & - & - & A \\
\hline FEB/FEA & 6 & 10 & - & - & - & 12 & 4 & A \\
\hline FEB/FEA & 13 & 4 & 4 & - & - & 1 & - & A \\
\hline FEB/CR/ & 1 & 7 & 17 & 9 & 4 & 5 & 11 & A \\
\hline $\begin{array}{c}\text { FEB/CR/ } \\
\text { FEA }\end{array}$ & - & - & 8 & 3 & 11 & 4 & 8 & A \\
\hline FEB/CR & - & 1 & 1 & - & 2 & - & - & A \\
\hline FEB & - & - & - & 1 & - & - & - & $\mathrm{R}$ \\
\hline CR/FEA & - & 1 & - & 1 & - & - & - & $\mathrm{R}$ \\
\hline $\begin{array}{c}\text { FEB/CR/ } \\
\text { FEA }\end{array}$ & 3 & 15 & 13 & 11 & 16 & 21 & 13 & $\mathrm{R} / \mathrm{A}$ \\
\hline FEB/CR & - & - & - & - & - & 2 & - & A \\
\hline $\begin{array}{c}\text { FEB/CR/ } \\
\text { FEA }\end{array}$ & - & 2 & 1 & 3 & 1 & 3 & 5 & A \\
\hline
\end{tabular}

CR

CR/FEA

CR

FEB/FEA

CR

CR

FEB 


\begin{tabular}{|c|c|c|c|c|c|c|c|c|c|c|}
\hline & \multirow{2}{*}{ Táxon } & \multirow{2}{*}{ TFA } & \multicolumn{7}{|c|}{ Indivíduos capturados por coleta } & \multirow{2}{*}{ MC } \\
\hline & & & Abr & Mai & Jun & Jul & Ago & Set & Out & \\
\hline \multirow{3}{*}{ Heliconiinae } & Euptoieta hegesia hegesia (Cramer) $\mathbf{c d}$ & CR & - & - & 2 & - & - & - & - & $\mathrm{R}$ \\
\hline & Heliconius erato phyllis (Fabricius) b $\mathbf{c} \mathbf{d e}$ & $\begin{array}{l}\text { FEB/CR/ } \\
\text { FEA }\end{array}$ & 1 & 2 & - & 2 & 3 & 1 & 1 & $\mathrm{R}$ \\
\hline & Heliconius ethilla narcaea (Godart) bed $\mathbf{e}$ & FEB/FEA & 1 & 2 & 3 & 2 & 4 & 1 & 3 & $\mathrm{R}$ \\
\hline \multirow{8}{*}{ Ithomiinae } & Dircenna dero rhoeo C. Felder \& R. Felder a c e & FEA & - & - & - & - & - & 2 & 2 & $\mathrm{R}$ \\
\hline & Episcada hymenaea hymenaea (Prittwitz) ${ }^{\mathbf{c d} \mathbf{e}}$ & FEB/FEA & 2 & - & 2 & 5 & 2 & 1 & 4 & $\mathrm{R}$ \\
\hline & Hypothyris euclea laphria (Doubleday) ${ }^{\mathbf{e}}$ & FEB & - & - & 3 & 1 & 1 & - & - & $\mathrm{R}$ \\
\hline & Melinaea ethra (Godart) ${ }^{\mathbf{e}}$ & FEB/FEA & - & - & - & 1 & 1 & 2 & - & $\mathrm{R}$ \\
\hline & Methona singularis (Staudinger) $\mathbf{c}$ & FEB/FEA & - & - & - & 1 & 3 & 1 & 1 & $\mathrm{R}$ \\
\hline & Pseudoscada erruca (Hewitson) & FEA & 2 & - & - & 1 & - & 1 & - & $\mathrm{R}$ \\
\hline & Scada reckia reckia (Hübner) ${ }^{\mathbf{c} \mathbf{d e}}$ & FEB & - & - & 1 & 1 & - & - & - & $\mathrm{R}$ \\
\hline & $\begin{array}{l}\text { Thyridia psidii cetoides (Rosenberg \& Talbot) } \\
\text { c }\end{array}$ & FEB & 1 & - & - & - & 1 & - & - & $\mathrm{R}$ \\
\hline Libytheinae & Libytheana carinenta carinenta (Cramer) ${ }^{\mathbf{c d}}$ & $\begin{array}{l}\mathrm{FEB} / \mathrm{CR} / \\
\text { FEA }\end{array}$ & - & 2 & 2 & 3 & - & - & 1 & $\mathrm{R}$ \\
\hline \multirow{2}{*}{ Limenitidinae } & Adelpha plesaure plesaure Hübner ${ }^{\mathbf{d e}}$ & FEA & - & 1 & - & 1 & - & 1 & - & $\mathrm{R}$ \\
\hline & Adelpha serpa serpa (Boisduval) •* & FEA & - & - & - & 1 & - & - & - & $\mathrm{R}$ \\
\hline Morphinae & $\begin{array}{l}\text { Opsiphanes cassiae crameri C. Felder \& R. } \\
\text { Felder b c }\end{array}$ & $\mathrm{CR}$ & - & 1 & - & - & - & - & - & A \\
\hline \multirow{7}{*}{ Nymphalinae } & Anartia jatrophae jatrophae (Linnaeus) a b c de & $\mathrm{CR}$ & - & - & - & 2 & - & - & - & $\mathrm{R}$ \\
\hline & Colobura dirce dirce (Linnaeus) b e e & FEB/FEA & - & 1 & 1 & - & - & - & 1 & A \\
\hline & Eresia lansdorfi (Godart) ${ }^{\mathbf{e}}$ & FEA & - & - & - & - & - & - & 1 & $\mathrm{R}$ \\
\hline & Historis odius odius (Fabricius) b c e & $\mathrm{CR}$ & - & - & - & - & - & - & 1 & A \\
\hline & Junonia evarete evarete (Cramer) ${ }^{\mathbf{b} \mathbf{c d e}}$ & $\mathrm{CR}$ & - & 1 & 4 & - & 1 & 1 & - & $\mathrm{R}$ \\
\hline & $\begin{array}{l}\text { Siproeta stelenes meridionalis (Fruhstorfer) }{ }^{\mathbf{b}} \\
\mathbf{c d \mathbf { e }}\end{array}$ & FEB/FEA & - & - & 1 & 2 & 2 & 1 & - & $\mathrm{R}$ \\
\hline & Tegosa claudina $(\text { Eschscholtz) })^{\mathrm{de}}$ & $\mathrm{CR}$ & - & - & - & - & - & 1 & - & $\mathrm{R}$ \\
\hline \multirow{8}{*}{ Satyrinae } & Euptychoides castrensis (Schaus) ${ }^{\mathrm{d}}$ & $\mathrm{CR}$ & 1 & - & - & - & 1 & 7 & 16 & $\mathrm{R} / \mathrm{A}$ \\
\hline & Hermeuptychia hermes (Fabricius) ${ }^{\mathbf{b} \mathbf{d} \mathbf{e}}$ & $\mathrm{CR}$ & - & - & - & - & - & 6 & 4 & A \\
\hline & $\begin{array}{l}\text { Pareuptychia ocirrhoe interjecta (d'Almeida) } \\
\text { cde }\end{array}$ & FEB & - & 1 & - & - & - & - & - & A \\
\hline & Magneuptychia libye (Linaeus) ${ }^{\mathbf{b}}$ & FEB/FEA & - & - & 1 & 1 & 1 & - & - & $\mathrm{R}$ \\
\hline & Taygetis laches (Fabricius) d e & FEB/FEA & - & - & - & - & 8 & 2 & 20 & A \\
\hline & Taygetis tripunctata Weymer ${ }^{\mathbf{e}}$ & FEB & - & 9 & 5 & 3 & 10 & 2 & - & A \\
\hline & Yphthimoides affinis (Butler) $\mathbf{e}^{\mathbf{2}}$ & FEA & 1 & - & - & - & - & - & - & $\mathrm{R}$ \\
\hline & Yphthimoides renata (Stoll) d e & $\begin{array}{l}\mathrm{FEB} / \mathrm{CR} / \\
\mathrm{FEA}\end{array}$ & 6 & 10 & 6 & 7 & 14 & 11 & 11 & $\mathrm{R} / \mathrm{A}$ \\
\hline \multicolumn{3}{|l|}{ TOTAL } & 74 & 110 & 141 & 89 & 110 & 114 & 118 & \\
\hline
\end{tabular}

principalmente nas áreas de Campo Rupestre, proveniente da ocorrência de chuva poucos dias antes da coleta. O oposto podese afirmar com relação à baixa riqueza no mês de Outubro em que, devido às condições climáticas desfavoráveis, quase não se observou a presença de recursos florais. Este dado é contrário ao apontado por Nobre et al. (2008) e ZaCCA \& Bravo (2012), que indicam uma maior diversidade de borboletas na Caatinga durante o período chuvoso (outubro a abril). Contudo, com os dados disponíveis até o momento, ainda não é possível afirmar qual ou quais fatores abióticos estariam influenciando nos padrões de riqueza e abundância das borboletas no Semiárido, sendo necessários mais estudos com este foco.

De acordo com Duarte JR. \& Schlindwein (2005) e Freitas \& Marins-Filho (2011), flutuações sazonais de disponibilidade de recursos podem acentuar a instabilidade de certas populações de lepidópteros na Caatinga. Entretanto, durante o período amostral, quatro espécies foram registradas em todos os meses de coleta: H. februa februa, Fountainea glycerium cratais (Hewitson), Heliconius ethilla narcaea (Godart) e Yphthimoides renata (Stoll). Por outro lado, 39 "singletons" (32\% do total) foram registrados, dos quais 19 pertencem à Hesperiidae (Tabela 1). Valores percentuais semelhantes de "singletons" também foram registrados por DESSUY \& Morais (2007) e GIOVENARdi $e t$ al. (2008). De acordo com Novotny \& BASSET (2000), vários fatores podem ocasionar a ocorrência de "singletons" nas amostragens, dentre eles o tamanho e sazonalidade das espécies difíceis de serem coletadas (BRown JR. \& FreITAS 1999).

A composição de espécies de borboletas se mostrou diferenciada ao longo do transecto percorrido, com 51 espécies (42,1\%) exclusivas de Campo Rupestre (com predominância de pierídeos e hesperídeos) e 43 (35,5\%) exclusivas de Floresta Estacional (Tabela 1). No entanto, as seguintes espécies foram comuns a ambas fitofisionomias: H. februa februa, $H$. feronia feronia, F. glycerium cratais, Heliconius erato phyllis (Fabricius), Libytheana carinenta (Cramer), Yphthimoides renata (Stoll) e Urbanus dorantes dorantes (Stoll). Também é possível notar diferenças na riqueza de espécies nos dois fragmentos de Floresta Estacional formados pela presença de uma escarpa (Figura 2), sendo 13 espécies exclusivas de baixas altitudes e 17 espécies exclusivas de elevadas altitudes (Tabela 1). Estes valores indicam que a fitofisionomia e a altitude podem ser fatores determinantes na distribuição das populações de borboletas e na complexidade dos nichos ecológicos de cada espécie, contudo são necessários mais estudos para maiores conclusões. 
Tabela 2. Riqueza de borboletas para o estado da Bahia.

\begin{tabular}{lcc}
\hline $\begin{array}{c}\text { Pesquisas realizadas no estado da } \\
\text { Bahia }\end{array}$ & No de espécies registradas & $\begin{array}{c}\text { No de espécies igualmente } \\
\text { registradas na Serra da Fumaça }\end{array}$ \\
\hline MAY (1924) & Não indica número exato & 3 \\
VASCONCELOS et al. (2009) & 70 & 32 \\
\hline ZACCA (2009) & 107 & 55 \\
ZACCA et al. (2011) & 140 & 60 \\
\hline ZACCA \& BRAVO (2012) & 169 & 67 \\
\hline
\end{tabular}

Novos registros para o Semiárido e estado da Bahia. Até o presente momento, 391 espécies de borboletas eram conhecidas para a região do Semiárido brasileiro (NoBre et al. 2008; ZACCA 2009; ZacCA et al. 2011; Paluch et al. 2011; Nobre et al. 2012; ZACCA \& BRAVo et al. 2012). A partir dos dados obtidos no presente estudo, mais 16 espécies foram incrementadas para o Semiárido (Tabela 1), correspondentes às famílias Hesperiidae (7 spp.), Nymphalidae (4 spp.), Riodinidae (3 spp.) e Pieridae (1 spp.).

Dos novos registros de Hesperiidae, destaca-se Panoquina peraea (Hewitson) conhecida apenas para o estado do Rio de Janeiro (Mielke \& CASAGRANDE 2002), que teve a sua distribuição geográfica ampliada mais ao norte, sendo registrada pela primeira vez tanto para o Semiárido quanto para a Bahia. Apesar de ser considerada endêmica do Cerrado por Bächtold (2010), Udranomia spitzi (Hayward) ocorre em áreas de campos das regiões sul, sudeste e centro-oeste do Brasil (DZUP), com seu primeiro registro para o Semiárido e Bahia. Narcosius parisi parisi (Williams) era conhecida para as regiões norte (estado de Rondônia e Pará), nordeste (Paraíba e Alagoas), centro-oeste (Mato Grosso) e sul (estados do Paraná e Santa Catarina) do Brasil (DZUP), tendo seu registro ampliado para o Semiárido e Bahia. Apesar de possuírem uma ampla distribuição geográfica no Brasil (DZUP), Orses cynisca (Swainson), Astraptes fulgerator fulgerator (Walch), Autochton integrifascia (Mabille) e Chiomara georgina georgina (Reakirt) são registradas pela primeira vez para o Semiárido e Bahia.

Quatro espécies de Nymphalidae são novos registros para o Semiárido e Bahia, Eunica eurota dolores (Prittwitz), conhecida apenas nas regiões sudeste e sul do Brasil (DZUP), Adelpha serpa serpa (Boisduval) e Actinote genitrix genitrix d'Almeida, com ocorrência nas regiões centro-oeste (Minas Gerais), sudeste e sul do Brasil (DZUP, Francini \& Penz 2006) e Hamadryas arinome obnubila (Fruhstorfer), conhecida para os estados de Pernambuco, Alagoas, Espírito Santo, Rio de Janeiro e Santa Catarina (DZUP).

As espécies Emesis fatimella fatimella Westwood, Synargis galena (Bates) e Nymphidium lisimon attenuatum Stichel são novos registros tanto para o Semiárido quanto para a Bahia. $S$. galena era conhecida apenas para a região amazônica (DZUP, Penz \& DeVries 1999), enquanto que $N$. lisimon attenuatum apenas para os estados de Goiás, Espírito Santo e São Paulo (DZUP). O pierídeo Rhabdodryas trite trite (Linnaeus) é um novo registro para o Semiárido, porém apresenta uma ampla distribuição no Brasil (DZUP).

Dos inventários (MAY 1924; VASCONCELOS et al. 2009; ZACCA \& BRAVO 2012) e listagens (ZACCA 2009; ZACCA et al. 2011) realizados na Bahia, o que registrou maior número de espécies e similaridade faunística com a Serra da Fumaça foi o de ZACCA \& BrAvo (2012), com 67 espécies em comum (Tabelas 1 e 2). Destaca-se que a própria Serra da Fumaça foi um dos pontos de amostragem no estudo de ZACCA \& Bravo (2012), onde capturaram 82 espécies, das quais 47 foram igualmente registradas no presente trabalho. Subtraindo as espécies em comum, obtém-se um total de 157 espécies para a região, das quais três são novos registros para a Bahia: Saliana longirostris (Sepp), Chioides catillus (Cramer), Epargyreus socus (Hübner).
O baixo número de espécies de borboletas registradas para a Semiárido, bem como para o estado da Bahia, indicam a necessidade de mais estudos a longo prazo nestas áreas, para obtenção de resultados satisfatórios quanto a diversidade de borboletas e realização de maiores inferências biológicas, já que fatores como distribuição horizontal, vertical e temporal podem influenciar na detecção das espécies (DEVRIES et al. 1997; DEVRIES \& WALLA 2001). Além de amostragens exaustivas, sugere-se a utilização de uma maior quantidade de armadilhas, dispostas em vários extratos da vegetação para uma melhor avaliação da diversidade local de borboletas, em futuros estudos.

\section{AGRADECIMENTOS}

Os autores agradecem ao Dr. André Victor Lucci Freitas (UNICAMP) pelo fornecimento de bibliografia especializada; Dr. Robert K. Robbins (National Museum of Natural History, Smithsonian Institution, Washington, D. C., USA) e Dr. Curtis J. Callaghan (Bogotá, Colombia) pelo auxílio com a identificação dos Nymphalidae, Lycaenidae e Riodinidae, respectivamente, ao MSc. Diego R. Dolibaina (UFPR) pela identificação dos Hesperiidae. Ao Dr. Eduardo Carneiro (UFPR) e aos dois referis anônimos da Entomobrasilis pela revisão do manuscrito, bem como as valiosas sugestões e comentários. Á Rangel Batista de Carvalho pela disponibilização do mapa da trilha da Serra da Fumaça.

\section{REFERÊNCIAS}

Bächtold, A., 2010. Ecologia das interações entre Udranomia spitzi (Lepidoptera, Hesperiidae) e formigas tendo como hospedeira Ouratea spectabilis (Ochnaceae) em um Cerrado mineiro. Dissertação (Mestrado em Ecologia e Conservação de Recursos Naturais) - Universidade Federal de Uberlândia. 33p.

Brown Jr., K.S. \& A.V.L. Freitas, 1999. Lepidoptera, p. 225-245. In: Brandão, C.R.F. \& E.M. Cancello (Eds.). Biodiversidade do Estado de São Paulo, Brasil. Invertebrados Terrestres. FAPESP, São Paulo, 279p.

Cowell, R.K., 2006. EstimateS - Statistical estimation of species richness and shared species from samples. Versão 8.2. Disponível em: <http://viceroy.eeb.uconn.edu/estimates $>$.

Dessuy, M.B. \& A.B.B. Morais, 2007. Diversidade de Borboletas (Lepidoptera, Papilionoidea e Hesperioidea) em fragmentos de floresta estacional decidual em Santa Maria, Rio Grande do Sul, Brasil. Revista Brasileira de Zoologia, 24: 108-120.

DeVries, P.J., D. Murray, R. Lande, 1997. Species diversity in vertical, horizontal, and temporal dimensions of a fruitfeeding butterfly community in an Ecuatorian Rainforest. Biological Journal of the Linnean Society, 62: 343- 364.

DeVries, P.J.\&T.R. Walla, 2001. Species diversity and community structure in neotropical fruit-feeding butterflies. Biological Journal of the Linnean Society, 74: 01-15.

Duarte Jr., J.A. \& C. Schlindwein, 2005. Riqueza, abundância e sazonalidade de Sphingidae (Lepidoptera) num fragmento de Mata Atlântica de Pernambuco, Brasil. Revista Brasileira de Zoologia, 22: 662-666.

Francini,R.B.\&C.M.Penz, 2006.AnillustratedkeytomaleActinote from Southeastern Brazil (Lepidoptera, Nymphalidae). Biota Neotropica 6: BNo0606012006. < http://www. 
biotaneotropica.org.br/v6n1/pt/abstract?identificationkey+bno0606012006>.

Freitas, A.V.L., R.B. Francini \& K.S. Brown Jr., 2003. Insetos como indicadores ambientais, p. 125-15. In: Cullen Jr. \& L., R. Rudran, C. Valladares-Padua (Eds.) Métodos de estudo em biologia da conservação e manejo da vida silvestre. Curitiba, Ed. Universidade Federal do Paraná, 652p.

Freitas, A.V.L. \& O.J. Marini-Filho, 2011. Plano de ação nacional para a conservação dos Lepidópteros. Brasília, Instituto Chico Mendes de Conservação da Biodiversidade (ICMBio), 124p.

Giovenardi, R., R.A. Di Mare, J. Sponchiado, S.H. Roani, F.A.F. Jacomassa, A.B. Jung \& M.A. Porn, 2008. Estudo da diversidade de borboletas (Lepidoptera: Rhopalocera) em duas localidades no município de Frederico Westphalen, Rio Grande do Sul, Brasil. Revista Brasileira de Entomologia, 52: 599-605.

Lamas, G., 2004. Checklist: Part4A, Hesperioidea-Papilionoidea, 1-439. In: Heppner, J. (Ed.). Atlas of Neotropical Lepidoptera. Association for Tropical Lepidoptera, Scientific Publishers, 479p.

May, E., 1924. Relatório das excursões effectuadas nos estados do Rio, Minas Gerais e Bahia. Boletim do Museu Nacional, 1: $367-375$.

Ministério do Meio Ambiente (MMA), 2007. Áreas prioritárias para conservação, uso sustentável e repartição dos benefícios da biodiversidade brasileira: Caatinga. Brasília, Ministério do Meio Ambiente (MMA), 292 p.

Mendes, B.V., 1994. Uso e conservação da biodiversidade do Semi-Árido. In: Ministério da Integração Nacional (Brasil). Projeto Áridas: recursos naturais e meio ambiente, 56p.

Mielke O.H.H. \& M.M. Casagrande, 1997. Papillonoidea e Hesperioidea (Lepidoptera) do parque estadual do Morro do Diabo, Teodoro Sampaio, São Paulo, Brasil. Revista Brasileira de Zoologia, 14: 967-1001.

Mielke, O.H.H. \& M.M. Casagrande, 2002. Notas taxonômicas em Hesperiidae neotropicais, com descrições de novos taxa (Lepidoptera). Revista Brasileira de Zoologia, 19: 27-76 (Suplemento).

Nobre, C.E.B., C. Schlindwein \& O.H.H. Mielke, 2008. The butterflies (Lepidoptera: Papilionoidea and Hesperioidea) of the Catimbau National Park, Pernambuco, Brazil. Zootaxa, 1751: 35-45.

Nobre, C.E.B., L. Iannuzzi \& C. Schlindwein, 2012. Seasonality of fruit-feeding butterflies (Lepidoptera, Nymphalidae) in a Brazilian Semiarid Area. International Scholarly Research Network Zoology, 2012: 1-8.

Novotny, V. \& Y. Basset, 2000. Rare species in communities of tropical insect herbivores: pondering the mystery of singletons. Oikos, 89: 564-572.

Paluch, M., O.H.H. Mielke, C.E.B. Nobre, M.M. Casagrande, D.H.A. Melo \& A.V.L. Freitas, 2011. Butterflies (Lepidoptera: Papilionoidea and Hesperioidea) of the Parque Ecológico
João Vasconcelos Sobrinho, Caruaru, Pernambuco, Brazil. Biota Neotropica, 11: 229-238.

Penz, C.M. \& P.J. DeVries, 1999. Preliminary assessment of the tribe Lemoniini (Lepidoptera: Riodinidae) based on adult morphology. American Museum Novitates, 3284: 1-32.

Programa de Pesquisa em Biodiversidade do Semiárido (PPBio), 2011. Disponível em: <http://www.uefs.br/ppbio > Acesso em: dezembro 2011.

Ramalho, C.I., 2008. Estrutura da vegetação e distribuição espacial do licuri (Syagrus coronata (Mart) Becc.) em dois municípios do Centro Norte da Bahia, Brasil. Tese (Doutorado em Agronomia) - Universidade Federal da Paraíba. 131p.

Roman, M., J. Garlet \& E.C. Costa, 2010. Levantamento populacional e comportamento de voo de borboletas (Lepidoptera) em um remanescente florestal em São Sepé, RS. Ciência Florestal, 20: 283-294.

Santos, E.C., O.H.H. Mielke \& M.M. Casagrande, 2008. Inventários de borboletas no Brasil: estado da arte e modelo de áreas prioritárias para pesquisa com vistas à conservação. Natureza \& Conservação, 6: 68-90.

Uehara-Prado, M., K.S Brown \& A.V.L. Freitas, 2007. Species richness, composition and abundance of fruit-feeding butterflies in the Brazilian Atlantic Forest: comparison between a fragmented and continuous landscape. Global Ecology and Biogeography, 16: 43-54.

Vale, M.F.S, 2005. Análise da percepção dos moradores da Serra de Jacobina em relação ao desenvolvimento do ecoturismo associado à educação ambiental. Dissertação (Mestrado em Ecologia e Biomonitoramento) - Universidade Federal da Bahia. 135p.

Vasconcelos, R.N., E.C.C. Barbosa \& M.C.L Peres, 2009. Borboletas do parque metropolitano de Pituaçu, Salvador, Bahia, Brasil. Sitientibus, 9: 158-164.

Velloso, A.L., V.S.B. Sampaio \& F.G.C. Pareyn, 2002. Ecorregiões propostas para o bioma caatinga. Recife, APNE / Nature Conservancy do Brasil, 8op.

Zacca, T., 2009. Espécies de borboletas (Lepidoptera: Papilionoidea e Hesperioidea) da coleção entomológica Prof. Johann Becker do Museu de Zoologia da Universidade Estadual de Feira de Santana, Bahia, Brasil. Sitientibus, 9: 165-173.

Zacca, T., F. Bravo \& M.X. Araújo, 2011. Butterflies (Lepidoptera: Papilionoidea and Hesperioidea) from Serra da Jibóia, Bahia State, Brazil. EntomoBrasilis, 4: 139-143.

Zacca, T.\&F. Bravo, 2012. Borboletas (Lepidoptera: Papilionoidea e Hesperioidea) da porção norte da Chapada Diamantina, Bahia, Brasil. Biota Neotropica, 12: 01-10.

\section{Recebido em: 10/05/2013}

Aceito em: 26/09/2013

\section{Como citar este artigo:}

Lima, J.N.R. \& T. Zacca, 2014. Borboletas (Lepidoptera: Hesperioidea e Papilionoidea) de uma Área de Semiárido na Região Nordeste do Brasil. EntomoBrasilis, 7 (1): 33-40.

Acessível em: doi:10.12741/ebrasilis.v7i1.351
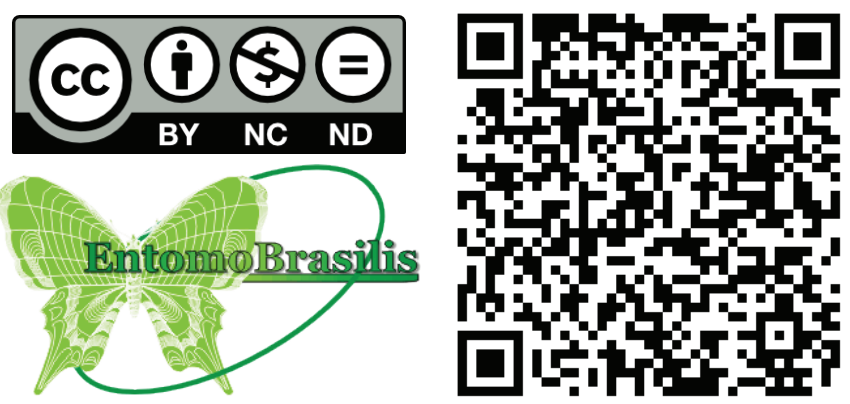